\title{
Engineering Design Optimization by Dynamic Cluster based Framework using Mixture Surrogates
}

\author{
Shailesh Sharad Kadre, Vipin Kumar Tripathi
}

\begin{abstract}
The real-world engineering optimization problems utilize complex computational methods like finite element frameworks. These approaches are computationally costly and need high solution time. The work pays attention on finding the optimal solution to these complex engineering problems by using Surrogate Models (SM). SMs are mathematical models, which are utilized to minimize the required number of such costly function evaluations at the time of the optimization cycles. Instead of optimizing the Design Space (DS) as a whole, subregion based strategies are found to be effectual, especially in the cases where prior knowledge of optimal solution is unavailable. In the present work, a surrogate centered optimization scheme is presented for local search, which dynamically sub-divides the DS into an optimum number of sub-regions by choosing the best cluster evaluation techniques as followed by the selection of best mixture SMs for each optimization cycle. For all objective functions and constraint functions in every sub-region, the mixture SMs are created by a combination of two or more single SMs. The MATSuMoTo, the Matlab based SM Toolbox by Juliane Muller and Robert Piché has been adapted for the creation and selection of best mixture SM. In this method, an individual surrogate is combined by utilizing the DempsterShafer theory (DST). Besides the above local search, a global search module is also introduced for ensuring faster convergence. This approach is tested on a constrained optimization benchmark test problem with smaller, disconnected feasible regions. It is perceived that the proposed algorithm accurately located all the local and global optima points with minimum function evaluations. The approach is applied to engineering problems like optimization of Machine Tool Spindle (MTS) design and frontal crash simulation on a full car body. For these engineering application problems also, mixture SMbased sub-region based search strategy is utilized to attain most accurate global optimum solution with a minimal number of costly function evaluations.
\end{abstract}

Keywords: Machine tool spindle design, Crash simulation, Mixture surrogate models, Sub-regions, Genetic algorithm.

\section{INTRODUCTION}

Today, Computer simulations are utilized to approximate the complex physical behaviours like a car crash and durability simulations. Those simulations are usually computationally much expensive, and hence during optimization, the objective is to ascertain optimal solutions by using a restricted number of evaluations of those expensive functions. SMs are utilized for approximating the

Revised Manuscript Received on September 10, 2019. Engineering, College of Engineering, Savitribai Phule Pune University, Maharashtra, India.

(Email id: shaileshkadre@gmail.com)

Dr. Vipin Kumar Tripathi, Department of Mechanical Engineering, College of Engineering, Savitribai Phule Pune University, Maharashtra, India.

(Email id: vkt.mech@coep.ac.in)
Shailesh Sharad Kadre, Research Scholar, Department of Mechanical

costly simulation model. Grounded on the predictions made by SMs, new infill points are created in the DS where the expensive objective functions are to be assessed. This approach diminishes the computation time of this optimization considerably owing to less expensive function evaluations. Some instances of interpolating SMs are Kriging and Radial basis functions (RBF). Polynomial regression models of various orders are non- interpolating SMs.

Identification of the exact location of multiple local optima and global optima is conveniently found by decomposing the whole DS into several sub-regions and then solving each sub-region centered local optimization. Diane Villanueva et al [1,2] showed that using a system of sub-division based surrogate-centric optimization strategy is more effective at locating the global and local optima as compared to optimization with a single surrogate over the whole DS. In literature, there are innumerable methods of DS partitioning. The related studies by authors [3] showed a scheme of SM based scheme applied on sub-region techniques which use only single best SM for the predetermined number of sub-regions.

It is an eminent fact that a single-SM might not be best suitable for all sorts of problems. This resulted in a concept of selection of best single model or the best mixture of individual surrogate for given engineering application problem. An approach was introduced by T. Goel [3] for combining the individual SM had limitations of adjusting the un-known parameter prior to the start of the optimization cycle. This sometimes may result in individual weights greater than one or even negative too. All the above limitations may bring inaccuracy in the final results. In order to overcome the aforementioned problems the MATSuMoTo, the Matlab based SM Toolbox by Juliane Muller and Robert Piché [5] has been adapted for the creation and selection of the best mixture SM. In this method, an individual surrogate is combined by using DST [5]. This method does not need fine-tuning of any parameters beforehand and allows the combination of even conflicting and inaccurate characteristics of individual SMs to be used for mixture SM. In this work, instead of considering just one model characteristics for best SM, four model characteristics are calculated for every SM. These characteristics are correlation coefficients, root-meansquared errors, maximum absolute errors, and median absolute deviation. Except for correlation coefficients, the other three low-valued parameters are preferred. So it is 
evident that these model characteristics are conflicting in nature. In such cases where it requires a combination of various model characteristics, DST is found effective (Juliane Muller [5]) by calculating the Basic Probability Assignment (BPA). Grounded upon these BPAs, best SM is chosen amongst all available models.

In a former study [7], authors have presented surrogatecentric design optimization for various engineering applications using the Kriging model. Tripathi et al. [8-10] studied the optimization of real engineering problems like multi-objective optimization studies on gearbox, shape by shape rigid-body docking, etc. SM was not used in these studies for optimization. The strategy of using the best mixture SM with sub-region based optimization is not available to the best of author's knowledge. For handling constraints, the Augmented Lagrangian Genetic Algorithm (ALGA) [11] is used instead of the Penalty method as originally suggested in MATSuMoTo Toolbox [5].

The remaining parts of this paper are provided in the succeeding sections: Section 2 details the algorithm utilized for dynamic partitioning based constrained optimization using mixture surrogates. Section 3 illustrates the algorithm with an example benchmark problem along with the concept of dynamic partitions of DS. Sections 4 provide a clear explanation of numerical experiments done for validating the developed algorithm along with its computer implementation. In Section 5, the introduced techniques are applied to a benchmark test problem and engineering problems include frontal crash simulation on the car model problem and design of MTS.In Section 6, conclusion and also scope of future studies are provided.

\section{DYNAMIC PARTITIONING BASED CONSTRAINED OPTIMIZATION (DPBCO) USING MIXTURE SURROGATES ALGORITHM}

This section provides the details of the sub-region-based algorithm utilized for the surrogate-aided global optimization problems. Sub-region centered approach starts with the run- time evaluation of number of clusters of DS followed by determination of centroid of all sub-regions. Usually, the centroid is the sub-region minima existent in the previous optimization cycle. In absence of feasible centroid, (sub-regions minima which follows all the constraints) centroid is the geometrical center determined by $\mathrm{k}$ - means clustering (KMC) algorithms. In each optimization cycle, it is ensured that the newly added optimum solution is not duplicate and generated on already dense area. In these situations, a new point is generated by maximizing the minimal distance of this point from the already existing ones. The Algorithm 1 is implemented in the succeeding steps as follows:

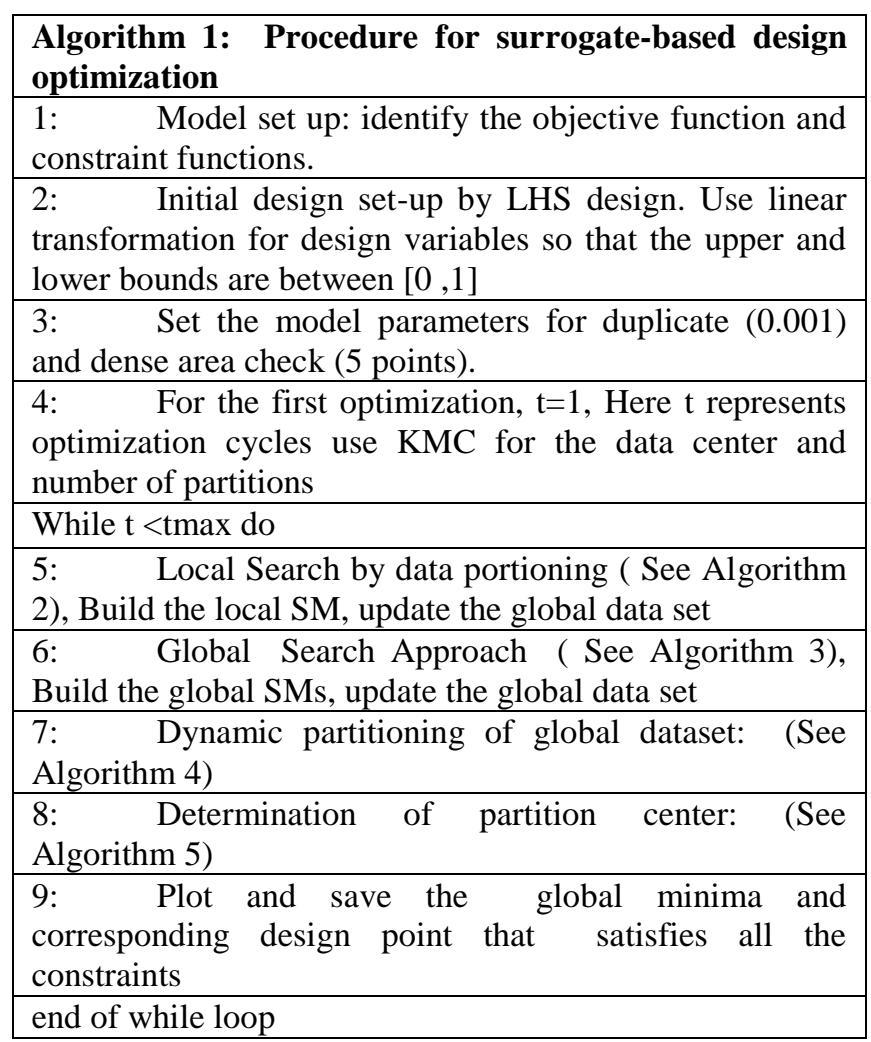

In the Model setup, the objective functions and constraint functions are recognized. Find the initial set of sample points using, for example, LHS design, and evaluate costly objective functions and constraint functions at those points. Decide the initial point tolerance (duplicate check 0.001) and , cluster tolerance ( dense area check- 0.05 , points $>5$ in the tolerance zone is dense area). While $(t=1)$, the initial stage use KMC to partition the design stage and find the center of each partition. Here $t$ represents the optimization cycle. 


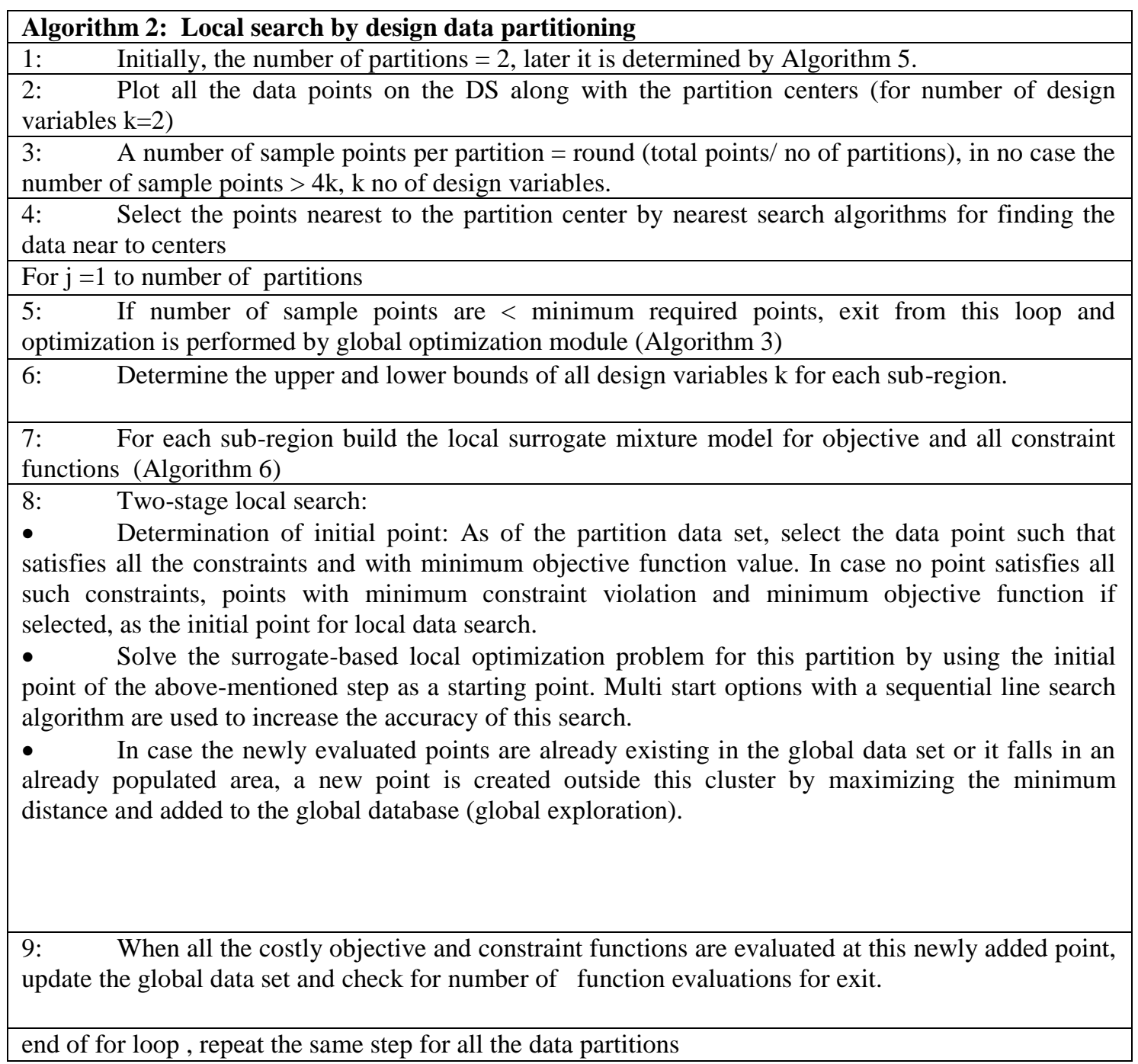

In the beginning of the optimization algorithm, very few data points are available at each sub-region to build the best mixture SM. Here, the global strategy has been found useful till the global search shows no improvements. The subsequent Algorithm 3 elucidates the Global search approach.

\begin{tabular}{|c|}
\hline 10. \\
\hline $\begin{array}{l}\text { 1: Build the global SM of objective and } \\
\text { constraint functions from the global data set. Use } \\
\text { global upper and lower bounds for all the design } \\
\text { variables }\end{array}$ \\
\hline $\begin{array}{l}2: \quad \text { Add new sample sites by constrained } \\
\text { global optimization by using Genetic Algorithm }\end{array}$ \\
\hline $\begin{array}{l}\text { 3: For the new point, check for duplicate } \\
\text { and local cluster area check in respect of already } \\
\text { evaluated points }\end{array}$ \\
\hline $\begin{array}{l}\text { 4: Evaluate the costly objective function at } \\
\text { the new sample site(s) and update the global data } \\
\text { base for the objective and also constraint } \\
\text { functions. Update the global dataset }\end{array}$ \\
\hline $\begin{array}{l}\text { 5: Evaluate the new database for a new } \\
\text { number of partitions and find the new data } \\
\text { centers ( see methods of dynamic partitioning } \\
\text { and new data centers) }\end{array}$ \\
\hline
\end{tabular}

Following Algorithm 4 proffers the details of dynamic partitioning of a global dataset:

\begin{tabular}{l} 
Algorithm 4: Dynamic partitioning of a global dataset: \\
\hline 1: This step is applied for more than ' 5 ' optimization \\
cycles. For the first 5 design cycles, the number of clusters \\
is fixed to 2 to have an adequate number of data points \\
required for further dynamic partitioning. \\
\hline 2: Grounded on the characteristic of data points (for \\
example Euclidian distance within and amongst data points \\
of a cluster), the best cluster evaluation method is chosen for \\
an optimal number of clusters \\
\hline 3: The best available method from the available \\
cluster methods is selected. These methods are \\
CalinskiHarabasz Evaluation, Silhouette Evaluation, Gap \\
Evaluation, and DaviesBouldin Evaluation.
\end{tabular}


Following Algorithm 5renders the details of Determination of partition center

\begin{tabular}{|c|}
\hline \\
\hline $\begin{array}{l}\text { 1: After the determination of the optimum number } \\
\text { of partitions, the global design } \\
\text { partitioned by KMC. KMC also provides a sub-region } \\
\text { geometrical center. }\end{array}$ \\
\hline for $\mathrm{j}=1$ to number of partitions do \\
\hline $\begin{array}{l}\text { 2: Find the data set of design points that satisfy all } \\
\text { the constraints in the sub-region with corresponding } \\
\text { objective values. Find the point with minimal function } \\
\text { value from this data set [1]. The partition center is the } \\
\text { design point at this value of an objective function in the } \\
\text { partition dataset. }\end{array}$ \\
\hline $\begin{array}{l}\text { 3: If no points in a sub-region satisfy the } \\
\text { constraints, then the partition center is the center } \\
\text { determined by KMC algorithms. }\end{array}$ \\
\hline \\
\hline
\end{tabular}

\section{MIXTURE SURROGATE MODEL WITH DYNAMIC PARTITIONING: ILLUSTRATION}

This algorithm is illustrated with the behavior of the benchmark test function called the New Branin test function [1], which has two variables. This is a two-dimensional optimization problem with disconnected feasible regions that cover approximately $3 \%$ of the DS, as evinced in Figure. 1.

$\min$ imize $_{x} f(x)=-\left(x_{1}-10\right)^{2}-\left(x_{2}-15\right)^{2}, x_{1} \in[-5,10], x_{2} \in[0,15]$

Subjected to,

$$
g(x)=\left(x_{2}-\frac{5.1}{4 \Pi^{2}} x_{2}+\frac{5}{\Pi} x_{1}-6\right)^{2}+10\left[\left(1-\frac{1}{8 \Pi}\right) \cos x_{1}\right]
$$

In this study; the ranges of design variables are scaled in Eq.(1) in such wise that they vary betwixt $[0,1]$. In this example, both the objective function $\boldsymbol{f}$ and constraint $\boldsymbol{g}$ were considered to be expensive and were thus approximated with surrogates.

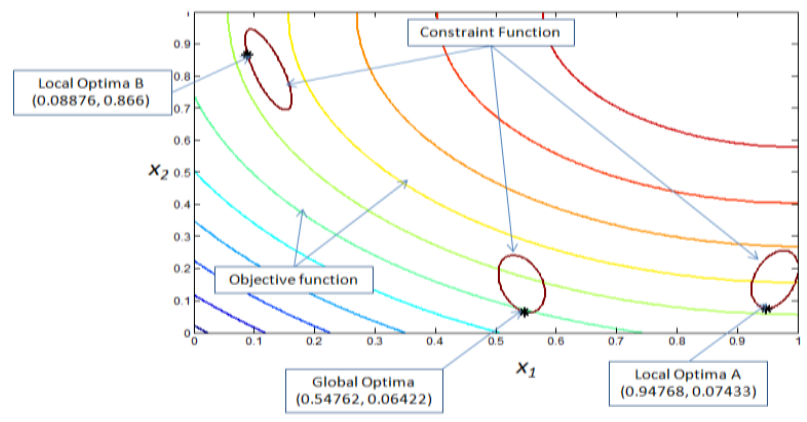

Figure.1: The new Branin test function[1]. The

function has one global optima and two local optima. The feasible region is disconnected

In Figure. 2, cycle 1 evinces the 2 clusters of initial random design of experiment (Experiment 20).
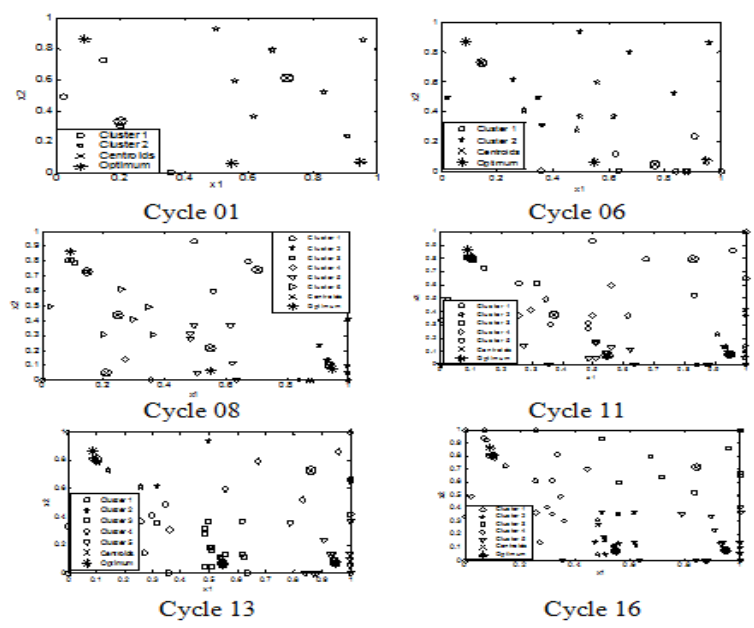

Figure. 2: 16 optimization cycles for new Branin test function for a random experiment (experiment number

20), shows the allocation of design points to dynamic partitions (clusters), (cluster centroids represented by a cross), and added points (optima are represented by stars).

For this, 12 initial points were created and clustering was done by KMC. The cross represents the geometric centers of these two clusters created by KMC algorithms. These centers are used as cluster centers as this is the first optimization cycle. The character '*' represents the actual optima of the constrained optimization cycle.

For the optimization cycles 3, 4, 5 and 6, the number of clusters is kept to 2 so that enough number of design points are available for making the mixture SMs after dynamic partitioning.

For optimization cycle 8 , the number of partitions changes to 6 and the solution points and partitions centroid move further closer to the local and also global optima. For cycle 9 , the global module switches to exploration mode due to duplicate and dense area conditions.

Again, Cycles 11 shows that in each cycle the global and local optima ' $\mathrm{A}$ ' are found accurately and respective cluster centroids coincide them. For local optima B, the accuracy within 0.05 distances is attained at cycle 16 .

\subsection{Runtime Determination of Optimum Number of Partitions in a Design Space}

There is no fixed method to determine the optimal number of clusters during the run-time of each optimization cycle. Figure. 3 elucidates the effect of changes of cluster evaluation method on the number of cluster per optimization cycle. On X-axis, number of optimization cycle is shown. Its $\mathrm{Y}$-axis displays the number of clusters using different cluster evaluation criteria.

From this Figure, it is perceived that the cluster evaluation criteria termed 'Gap criteria' shows a constant value of 2 for all 25 optimization cycles. This means that this criterion is unsuitable for partitioning the data set for the given problem. From this figure, it is also perceived that the Calinski-Harabasz criteria outperformed all the cluster evaluation methods for the given problem. 


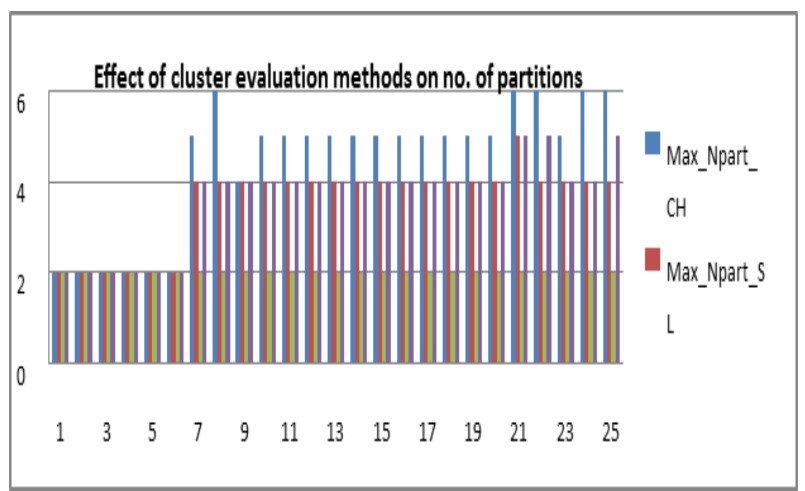

Figure. 3: Illustration of finding the optimum number of clusters for new branin test function for $\mathbf{4}$ different cluster evaluation algorithms using matlab inbuilt function evalclusters, sl- silhouette criteria, ch- calinskiharabasz criteria, db- davies-bouldin criteria and gpgap criteria.

\section{NUMERICAL EXPERIMENTS}

In this work, the chief objective is to contrast the performances proffered by the proposed algorithm of subregion based optimization with mixture surrogates versus the single SM based available approaches. The proposed algorithm is employed to the constrained optimization of benchmark function along with 2 real engineering applications. The succeeding sections clearly expound the numerical experiments performed in this work.

\subsection{Computer Implementation}

In this work, the MATSuMoTo (MATLAB SM Toolbox by Juliane Mullerand Robert Piché)is adapted [5] for the creation and selection of the best single and mixture SM. In this work, constraints were handled by selecting ALGA [11] to tackle nonlinear constraint problems. Here, the subregion-based algorithm is implemented with Matlab v20013b [11] on Dell®Inspiron 535S Core 2 Duo, 4GB RAM, Intel®processor

\subsection{Experimental Setup}

4.2.1. Analytical benchmark functions and engineering applications

An eminent analytical functionis utilized for testing the optimization algorithm. Following Table 1 elucidates these problems.

Table 1: Details of the benchmark and application problems

\begin{tabular}{lll}
\hline $\begin{array}{l}\text { Test and } \\
\text { Application } \\
\text { Problems }\end{array}$ & variables & Constraints \\
\hline $\begin{array}{l}\text { New Branin test } \\
\text { function[1] }\end{array}$ & 2 & 1 \\
$\begin{array}{l}\text { Optimization of } \\
\text { Machine tool }\end{array}$ & 4 & 4 \\
$\begin{array}{l}\text { spindle design[13] } \\
\text { Optimization of } \\
\text { car body under } \\
\text { the crash } \\
\text { constraints[14] }\end{array}$ & 4 & \\
\hline
\end{tabular}

4.2.2. Mixture surrogate model creation
MATSuMoTo, the Matlab based SM Toolbox by Juliane Muller and Robert Piché [5] is used for the creation and selection of best single and mixture SM. This MATSuMoTo is utilized as a framework for the current work which contains the library of various single and mixture SMs.

The following Table 2 evinces the details of SM considered in this study,

Table 2: Surrogates considered in this study [5]

\begin{tabular}{|c|c|}
\hline Single Model: & \\
\hline 'RBFcub' & Cubic RBF interpolant \\
\hline 'RBFlin' & Linear RBF interpolant \\
\hline 'POLYlin' & Linear regression polynomial \\
\hline 'POLYquad' & Full-quadratic regression polynomial \\
\hline 'POLYquadr' & $\begin{array}{l}\text { Reduced-quadratic } \\
\text { polynomial }\end{array}$ \\
\hline 'POLYcub' & Full-cubic regression polynomial \\
\hline 'POLYcubr' & Reduced-cubic regression polynomial \\
\hline $\begin{array}{l}\text { Mixture } \\
\text { Models: }\end{array}$ & \\
\hline 'MIX RcPc' & Ensemble of 'RBFcub' and 'POLYcub' \\
\hline 'MIX RcPcr' & Ensemble of 'RBFcub' and 'POLYcubr' \\
\hline 'MIX RcPq' & Ensemble of 'RBFcub' and 'POLYquad' \\
\hline 'MIX RcPqr' & $\begin{array}{l}\text { Ensemble of } \\
\text { 'POLYquadr' }\end{array}$ \\
\hline
\end{tabular}

The subsequent image proffers the details of the Best_Mix_Model_Stat funtion from MATSuMoTo [5] tool box.

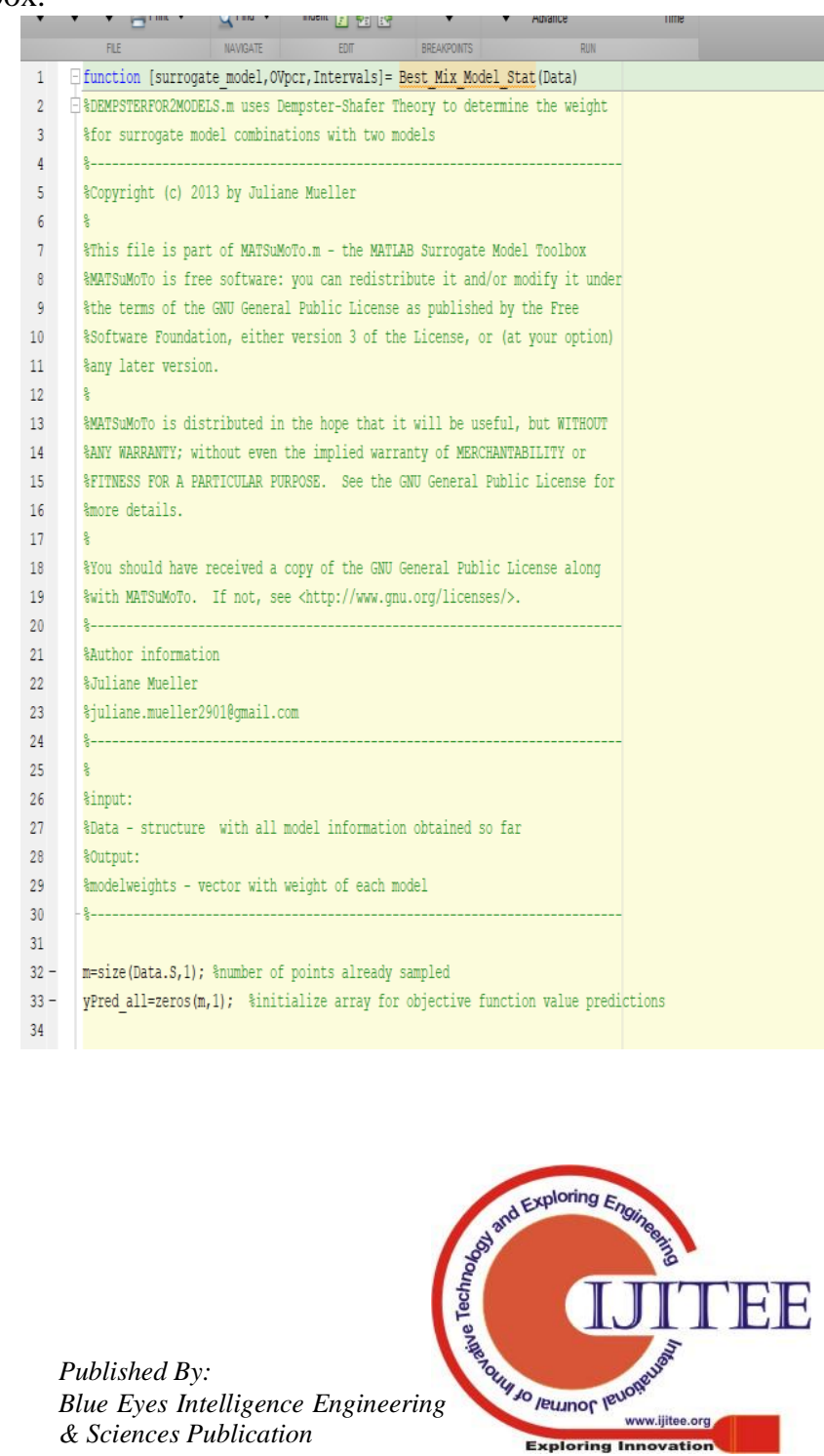




\subsubsection{Design of experiments and Setup for the proposed} algorithm

The quality of the approximation by meta-models and the rate of convergence to the optimum strongly depend on the number and distribution of the initial sampling points as defined in the DS (i.e., DOE). In the cases investigated, as a common practice in comparative studies of meta-modeling performance, repeat each experiment with 20 different DOE by using the Latin Hypercube Matlab function LHS design using default options.

The following Table 3 shows the details of considered process parameters.

Table 3:.Details of the parameters in this study

\begin{tabular}{ll}
\hline Parameter & Value \\
\hline Maximal number of partitions & 6 \\
Partitions before dynamic partitioning & 2 \\
Tolerance for cluster check & 0.05 \\
Min distance betwixt points ( Duplicate check) & $1.00 \mathrm{E}-03$ \\
Dynamic clustering after & 5 \\
Minimal \# of points in each agent after creation & 6 \\
Number of optimization cycles & 25 \\
Maximum number of points for local surrogates & 10 \\
\hline
\end{tabular}

\section{RESULT AND DISCUSSIONS}

\subsection{Benchmark Function (New Branin Test function)}

In this section, the effectiveness of present method for a complex benchmark function-New Branin Test function (as explained in section 3) is shown.

Table 4 evinces the number of times each surrogate is used over the cumulative number of iterations over 20 repetitions. From this table, it can also be perceived that for objective function, the utmost frequently utilized best mixture surrogate is the ensemble of MIX RcPc (POLYcub and RBFcub surface). For constraint functions, the utmost frequently utilized best SM is not common. Ensemble of MIX RcPc is utilized for constraints.

Table 4: Number of uses of each surrogate considering 20 repetitions

\begin{tabular}{lll}
\hline $\begin{array}{l}\text { Ensemble } \\
\text { Surrogate Model }\end{array}$ & $\begin{array}{l}\text { Objective } \\
\text { Functions }(\boldsymbol{f})\end{array}$ & $\begin{array}{l}\text { constraint } \\
\text { Functions }(g)\end{array}$ \\
\hline MIX RcPc & 2114 & 983 \\
MIX RcPcr & 352 & 478 \\
MIX RcPq & 107 & 561 \\
MIX RcPqr & 131 & 679 \\
\hline
\end{tabular}

Figure. 4 displays the success of locating a solution within some distance from the global optimum and other local optima with the best mixture models for all the strategies.20 successes were observed in all 20 repetitions of locating a solution within $3 \%$ of the global optimum and local optima A. Thus there were 16 successes for locating optima B within $10 \%$ distance from local optima B.

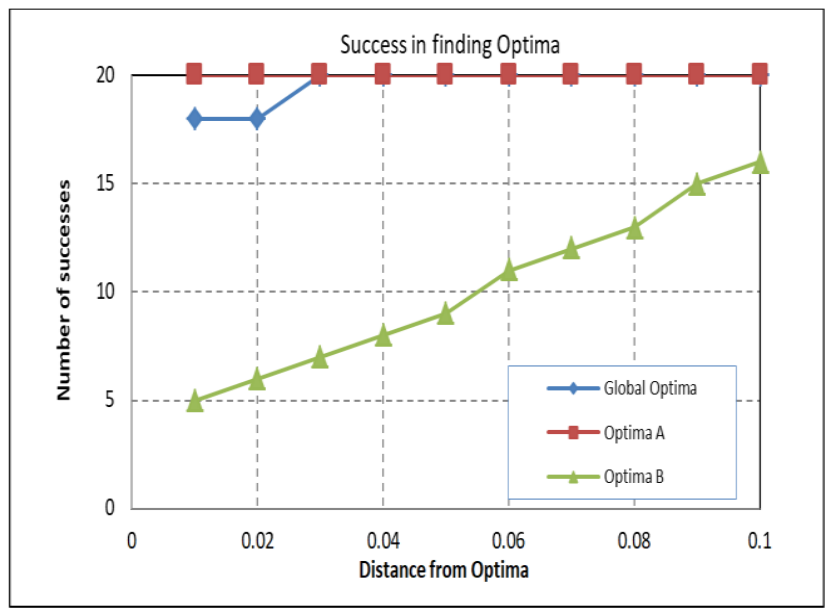

Figure 4: Number of successes Vs. distance from optima

Figure. 5 displays the median number of partitions through the 20 iterations. This Figure clearly shows that the number of partitions finally converged up to 6 . This is the prime reason that this method of partitioning could explore all the optima. For all the strategies in this problem, the Calinski-Harabasz criterion is used as the cluster evaluation method.

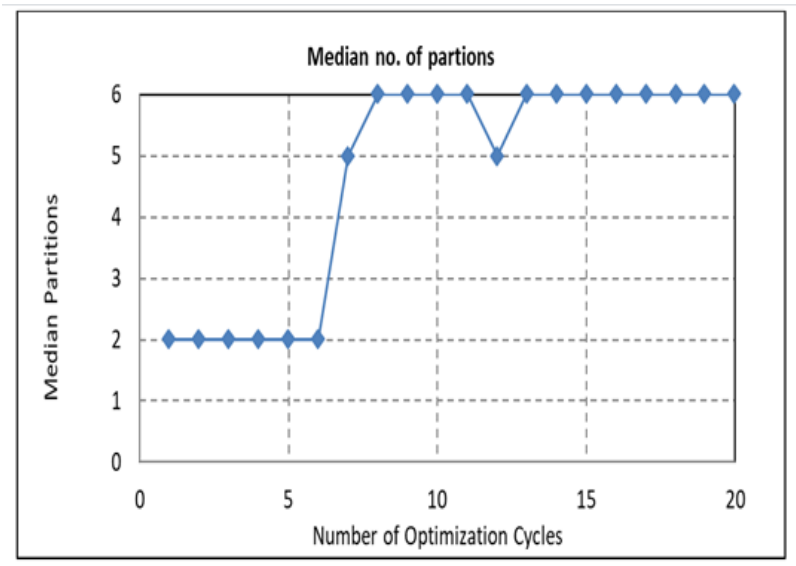

Figure 5: Median partition Vs number of Optimization cycles

Figure 6 delineates the Median distance of solutions nearglobal and local optima Vs median function evaluations. On contrasting the performance shown by the presented algorithm with the work performed by DianeVillanueva et al. [1] on the aforesaid function, succeeding observations could be made for $n=2$ initial partitions:

- Global optimum was found by the agent with the 29-function evaluations (compared to 40) within a distance of 0.008 from global optima.

- Local optima ' $A$ ' was found by the agent within 50-functionevaluations (compared to 70) within a distance of 0.015 from local optima A.

- Local optima 'B' was determined by the agent with the 45-function evaluations (compared to 60) within a distance of 0.05 from Local optima B. 


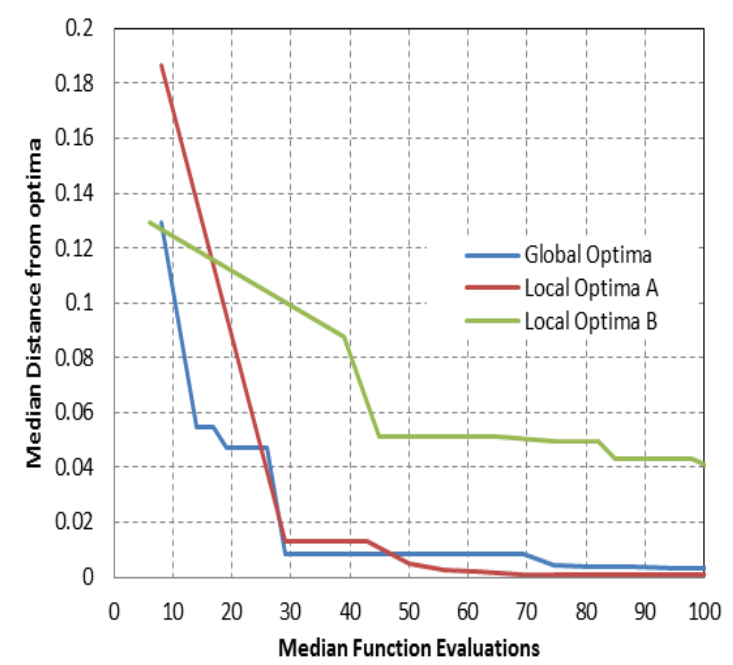

Figure 6: Median distance of solutions near-global and local optima Vs median function evaluations

Feasibility is not considered in finding the closest solution. The value ' $\mathrm{g}$ ' of the constraint function at this solution is evinced in Figure. 7. It perceived that the constraints began to satisfy after 14 function evaluations.

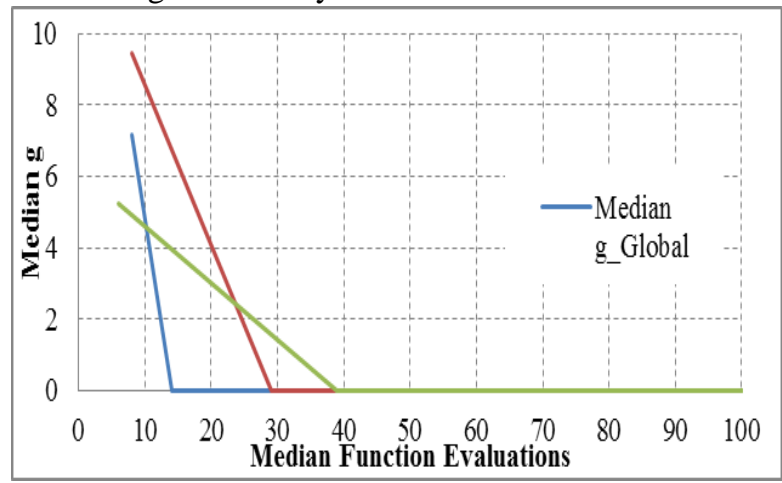

Figure 7: Median value of constraint g near-global and local optima

\subsection{Optimization of Machine Tool Spindle Design}

The aforesaid test problem is a practical applicationof this design[12]. Here, the actual problem is a bi-objective optimization problem (minimization of volumeas well as spindle deflection). A classical method suggested by Chankong and Haimes [15] is utilized to convert the biobjective optimization to single-objective optimization problem. The deflection objective function is converted to additional constraint by keeping $\in$ value as $0.018 \mathrm{~m}$ (Eq. 4). Eq. 5 and Eq. 6 show the design proportionality constraints. Eq. 7 symbolizes the spindle nose radial run-out. Eq. 8 provides the bound constraints of disparate geometrical parameters of this machine tool design.

Figure. 8 elucidates the typical MTS design with single objective function as a minimization of volume. The four design variables are $\boldsymbol{X}=\left\{\boldsymbol{l}, \boldsymbol{d}_{\boldsymbol{o}}, \boldsymbol{d}_{\boldsymbol{a}}, \boldsymbol{d}_{\boldsymbol{b}}\right\}$. Here $\boldsymbol{d}_{\boldsymbol{a}}$ and $\boldsymbol{d}_{\boldsymbol{b}}$ signifies thediscrete design variables that are assumed [13] to be taken from set $\boldsymbol{X}_{\mathbf{3}}=\{\mathbf{8 0}, \mathbf{8 5}, \mathbf{9 0}, \mathbf{9 5}\}$ and $X_{4}=\{75,80,85,90\}$ respectively.

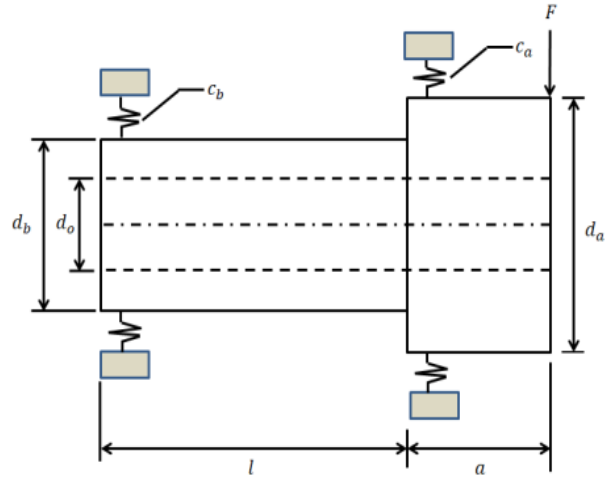

Figure 8: Adaptation of sketch of a typical MTS [13]

Objective function: Minimization of volume of spindle, $f_{5,1}(x)$

$$
\min \operatorname{imizef}(x)=\frac{\Pi}{4}\left[a\left(d_{a}^{2}-d_{0}^{2)}+\left(d_{b}^{2}-d_{0}^{2}\right)\right]\right.
$$

spindle deflection constraint, $g_{1}(x)$ subject to

$$
g_{1}(x)=\frac{F a^{3}}{3 E 1_{a}}\left(1+\frac{l I_{a}}{a I_{b}}\right)+\frac{F}{c_{a}}\left[\left(1+\frac{a}{l}\right)^{2}+\left(\frac{c_{a} a^{2}}{c_{b^{t^{2}}}}\right)\right]-\in \leq 0
$$

$$
I_{a}=0.049\left(d_{0}^{4}-d_{0}^{4}\right), I_{b}=0.049\left(d_{b}^{4}-d_{0}^{4}\right)
$$

Here,

Bearing Stiffness,

$c_{a}=35400\left|\delta_{r a}\right|^{\frac{1}{9}} d_{a}^{\frac{10}{9}}, c_{b}=35400\left|\delta_{b a}\right|^{\frac{1}{9}} d_{b}^{\frac{10}{9}}$

Design proportionality constraint, $g_{2}(x) ; g_{3}(x)$

$$
\begin{aligned}
& g_{2}(x)=p_{1} d_{0}-d_{b} \leq 0 \\
& g_{3}(x)=p_{2} d_{b}-d_{a} \leq 0
\end{aligned}
$$

Spindle nose

radial runout, $g_{4}(x)=\left|\Delta_{a}+\left(\Delta_{a}-\Delta_{b}\right) \frac{\alpha}{l}\right|-\Delta \leq 0$

Bound Constraints: $l_{k} \leq l \leq l_{g}, d_{a 2} \leq d_{a} \leq d_{a 1 \text {, }}$ $d_{b 2} \leq d_{b} \leq d_{b 1} ; d_{o m}-d_{o} \leq 0_{\mathrm{s}(8)}$

Following values of the parameters were assumed [13] $d_{o m}=25 \mathrm{~mm} ; d_{a 1}=80 \mathrm{~mm} ; d_{a 2}=95 \mathrm{~mm} ; d_{b 1}=75 \mathrm{~mm} ; p_{1}=1.25$;

$p_{2}=1.05 ; l_{k}=150 \mathrm{~mm} ; l_{g}=200 \mathrm{~mm}, a=80 \mathrm{~mm} ; E=210000 \mathrm{~N} / \mathrm{mm}^{2} ; F=10000 \mathrm{~N} ; \Delta_{a}=0.0054 \mathrm{~mm} ;$ $\Delta_{b}=0.0054 \mathrm{~mm}$

$\Delta=0.01 \mathrm{~mm} ; \delta_{r a}=0.001 \mathrm{~mm} ; \delta_{r b}=-0.001 \mathrm{~mm} ; \epsilon=0.018 \mathrm{~m}$

Table 5elucidates the total number of times each surrogate is used over the number of iterations. For objective function, constraint 1 and constraint 3 , the surrogate that most frequently used was the 'MIX RcPc'. For constraint 2 and constraint 4, 'MIX RcPqr' was used most frequently. 
Table 5: Number of uses of each surrogate considering 15 optimization cycles

\begin{tabular}{llllll}
\hline \multirow{2}{*}{$\begin{array}{l}\text { Surrogate } \\
\text { Model }\end{array}$} & $\begin{array}{l}\text { Objective } \\
\text { Function } f_{1}(x)\end{array}$ & \multicolumn{5}{c}{ Constraint } \\
\cline { 3 - 6 } & & Functions & \\
\cline { 3 - 6 } MIX RcPc & 406 & $g_{1}(x)$ & $g_{2}(x)$ & $g_{3}(x)$ & $g_{4}(x)$ \\
MIX & 330 & 354 & 304 & 328 & 143 \\
RcPcr & & & & & \\
MIX RcPq & 302 & 316 & 276 & 243 & 285 \\
MIX & 403 & 326 & 443 & 388 & 710 \\
RcPqr & & & & & \\
\hline
\end{tabular}

The median number of partitions through the 15 iterations is evinced in Figure.9. This figure corroborates that the number of partitions changes from 5 to 6 . Davies-Bouldin criterionis utilized for the cluster evaluation methodology in this problem.

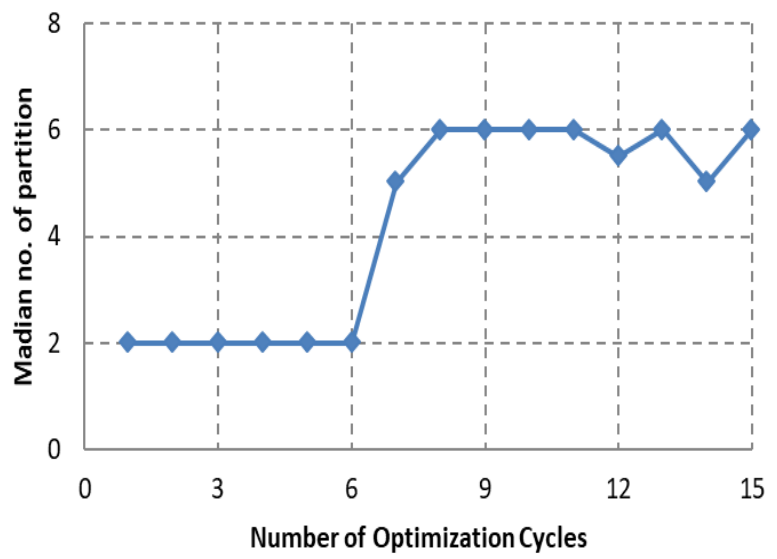

Figure 9: Median partitions vs number of optimization cycles

It is observed that the global optimum was found by the agent with the fewest number of function evaluations (approximately 60) as evinced in Figure. 10.

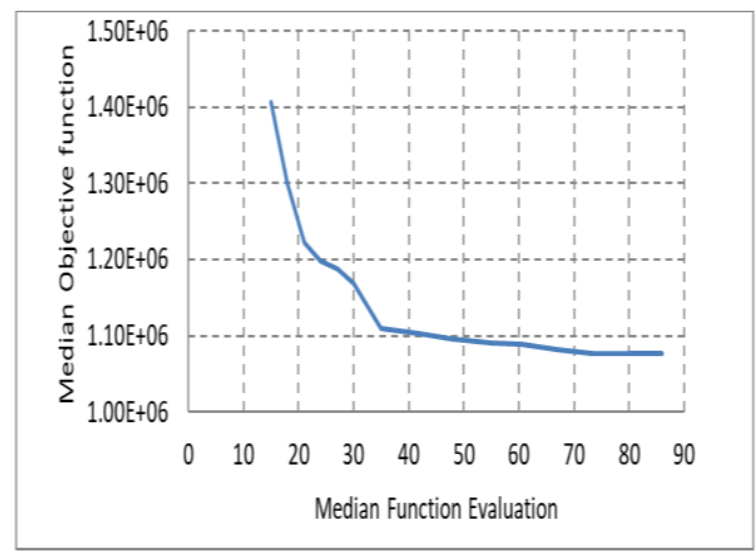

Figure 10: Median global optima Vs Median function evaluations

Feasibility is not considered in finding out the closest solution. The values of the constraint functions $\boldsymbol{g}_{\mathbf{1}}(\boldsymbol{x}), \boldsymbol{g}_{\mathbf{2}}(\boldsymbol{x}), \boldsymbol{g}_{\mathbf{3}}(\boldsymbol{x})$ and $\boldsymbol{g}_{\mathbf{4}}(\boldsymbol{x})$ at this solution areelucidatedusing Figure. 11.

This figure shows that the convergence of the solution is achieved with high accuracy.

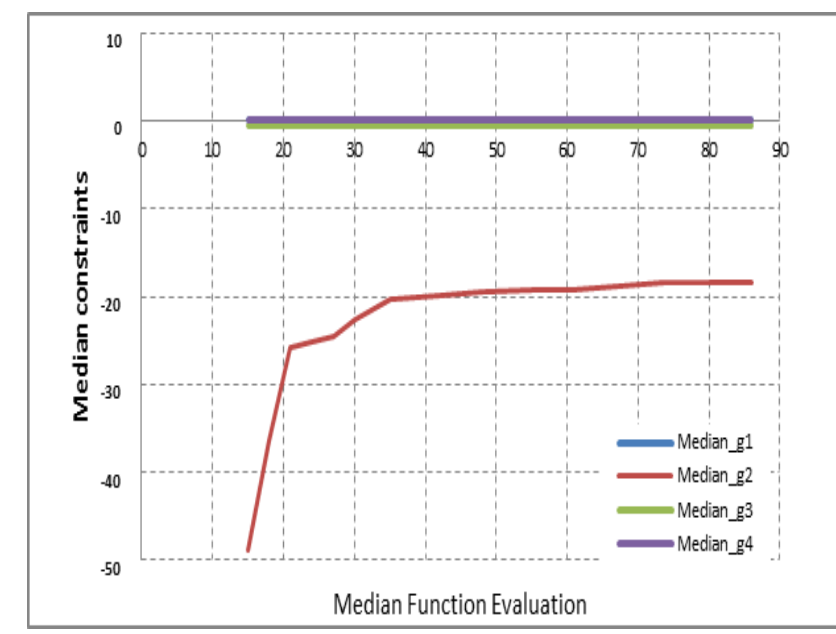

Figure 11: Value of constraints near global optima

\subsection{Optimization of Car Body Under Crash Constraints}

In this engineering application problem, crash simulations were considered as computationally expensive functions due to physical behavior and solution time. Liao et al. [14] studied the optimization framework for the crash safety model of vehicles by utilizing stepwise regression. This problem is originally a ' 3 ' objective problems which need to be studied: i) mass of vehicle, ii) integration of the collision acceleration betwixt $0.05 \mathrm{~s}$ and $0.07 \mathrm{~s}$ in "full frontal crash", and iii) toe board intrusions in "offset-frontal-crash" [14]. The variables are the thickness values of ' 5 ' reinforced members surrounding the frontal structures of the vehicles. This problem was transmuted in single-objective problem by classical method [15] for minimization of mass. Objective function of the integration of "full frontal crash" acceleration is converted to additional constraint by keeping $\in$ value as $7.5(\mathrm{~m} / \mathrm{s})$. Similarly for intrusion constraint, $\in$ was assumed to be $0.08 \mathrm{~m}$.

The median number of partitions through the 15 iterations is evinced in Figure. 12. This figure corroborates that the number of partitions remains constant at 6. A DaviesBouldin criterion is used for the cluster evaluation method for this problem.

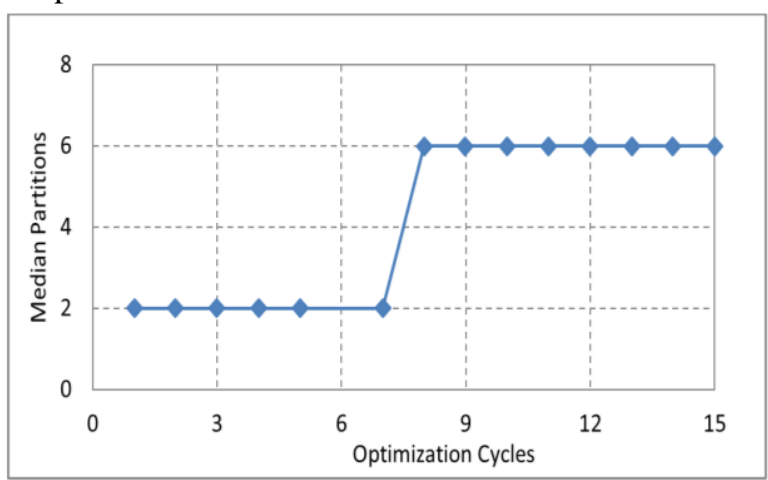

Figure 12: Median partitions Vs. number of optimization cycles 
Table 6 shows the total number of times each surrogate is used over the number of iterations. For objective function, the surrogate that utmost frequently used was the 'MIX RcPc'. For acceleration constraint function, 'MIX RcPc' was used most frequently. For intrusion constraint, 'MIX RcPc' was selected as the best SM.

Table 6: Number of uses of each surrogate considering 25 iterations

\begin{tabular}{llll}
\hline $\begin{array}{l}\text { Surrogat } \\
\text { e Model }\end{array}$ & $\begin{array}{l}\text { Objective } \\
\text { Functions }\end{array}$ & \multicolumn{2}{c}{ Constraint Functions } \\
\hline & \multicolumn{1}{c}{$f_{1}(x)$} & $g_{1}(x)$-Acc & $\begin{array}{l}g_{2}(x)- \\
\text { Intrusion }\end{array}$ \\
MIX & 740 & 770 & 764 \\
RcPc & & 181 & 198 \\
MIX & 219 & 236 & 231 \\
RcPcr & & & \\
MIX & 239 & 278 & 271 \\
$\begin{array}{l}\text { RcPq } \\
\text { MIX }\end{array}$ & 277 & & \\
RcPqr & & & \\
\hline
\end{tabular}

It is observed that the global optimum was found by the agent with the fewest number of function evaluations (approximately 37). Figure.13 displays the median global optima as a function of the median number of function evaluations.

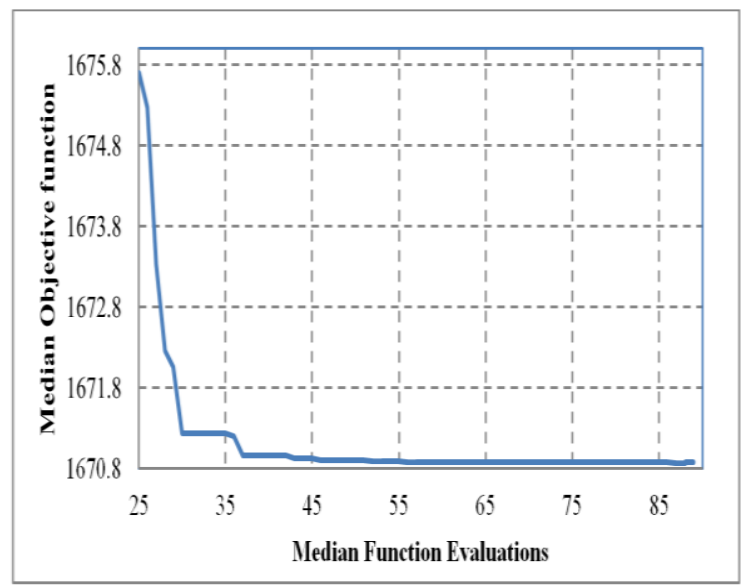

Figure 13: Median global optima vs median function evaluations

Feasibility is not considered in finding the closest solution. The values of the constraint function $\boldsymbol{g}_{\mathbf{1}}(\boldsymbol{x})$ and $\boldsymbol{g}_{2}(\boldsymbol{x})$ at this solution are evinced in Figure.14. The figures evinced that the convergence of solution is achieved with high accuracy.

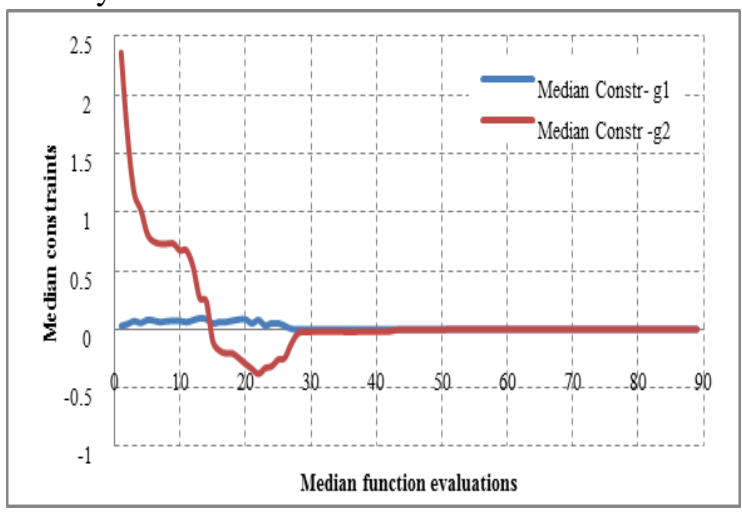

Figure 14: Median value of constraints near global optima

\section{CONCLUSIONS}

In this work, an approach for sub-region based optimization is presented using the best mixture SMs. The mixture models generated by means of DST for sub-regions and global models ensured both convergence and prediction quality during the whole optimization process.

Initially, the performance proffered by the proposed approach is delineated with two-dimensional analytical functions. It is observed that mixture SM and dynamic partitioning with a global module performs well for locating local and global optima with high accuracy and minimum number of function evaluation. The inclusion of global methods with sub-region based local searches makes this algorithm to work effectively.

The method properly works, both in the test problems and engineering applications with high accuracy and minimum number of function evaluations. This study shows the efficiency of the sub-region centered optimization techniques with effective handling of linear and non-linear constraints with a feasible number of costly function evaluation and optimization cycles. The DST helps to determine the best by choosing multiple model characteristics. This study elucidates the best SM changes in iterations and is different for objective functions and also constraint functions.

Although the attained results in the current work are in line with formerly published work in the related literature, a deep numerical and theoretical investigation, disparate functions of countless variables, use of different SMs for mixture models and increased number of partitions are is required to understand the behaviour and convergence features. The future work is to study their effect of the computational time and accuracy on the global optimization problems.

Funding

No Funding was available for this research.

\section{ACKNOWLEDGEMENTS}

We would like to thank the all the reviewers for their helpful comments.

\begin{tabular}{|ll|}
\hline Nomenclatures \\
$f(x)$ & Objective Function \\
$g_{i}(x)$ & Constraint functions, where $i$ is the constraint \\
$k$ & number \\
tmax & Number of design variables \\
$x$ & Maximum number of optimization cycles \\
Abbreviations & Aariable vector \\
ALGA & Designented Lagrangian Genetic Algorithm \\
DOE & Design space \\
DS & Dempster-Shafer Theory \\
DST & k- means clustering \\
KMC & Matlab based Surrogate Model Toolbox \\
MATSuMo & Machine Tool Spindle \\
To & Surrogate Model \\
MTS & \\
SM &
\end{tabular}




\section{REFERENCES}

1. Villanueva, D.; Riche, R.L.; Picard, G.; and Haftka, R.T.(2012a). Surrogate-based agents for constrained optimization. Proceedings of the 53rd AIAA/ASME/ASCE/AHS/ASC Structure, Structural Dynamics and Materials Conference. Honolulu, Hawaii.

2. Villanueva, D.; Riche, R.L.;Picard, G.; andHaftka, R.T.(2012b). Dynamic partitioning for balancing exploitation and exploration in constrained optimization: A multi-agent approach.Proceedings of the 14th AIAA/ISSMO Multidisciplinary Analysis and Optimization Conference. Indianapolis, IN.

3. Goel, T.; Haftka, R.T.; Shyy, W.; and Queipo, N.V. (2007). Ensemble of surrogates. Structural Multidisciplinary Optimization, 33(3), 199-216.

4. Mueller, J.(2014).MATSuMoTo.Retrieved March 10, 2016, fromhttps://arxiv.org/abs/1404.4261

5. Muller, J.; and Piché, R. (2011). Mixture surrogate models based on dempster-shafer theory for global optimization problems. Journal of Global Optimization, 51(1), 79-104.

6. Kadre, S.S.; and Tripathi, V.K. (2016). Advanced surrogate models for design optimization. Proceedings of World Congress on Engineering and Applications. Bangkok, Thailand.

7. Kadre, S.S.; and Tripathi, V.K. (2018). Constrained optimization by sub-region based best surrogate models. Proceedings of the First ASRES International Conference on Modeling, Analysis and Optimization. Amsterdam, Netherlands.

8. Tripathi,V.K.; Giri, M.; and Abhang, P.(2011). Optimum design of multi-speed gear box using genetic algorithm. Proceedings of the International Conference on Mechanical, Manufacturingand Industrial Engineering. Amsterdam, Netherlands.

9. Tripathi, V.K.; and Chauhan, H.M. (2010).Multiobjective optimization of planetary gear train.Lecture notes in computer science. Germany: Springer Verlag.

10. Tripathi, V.K.(2007). A patch-by-patch shape matching procedure for rigid body docking.Proceedings of the World Congress on Engineering.London,UK: Imperial College.

11. The MathWorks(2013). MATLAB 2013b. Natick, MA: The MathWorks, Inc.

12. Kalyan, S.B.; Hemant, K.S.; and Ray, T. (2016). Multiobjective optimization with multiple spatially distributed surrogates. Journal of Mechanical Design, 138(9), 1-10.

13. Tan, K.; Lee, T.; and Khor, E. (2001). Evolutionary algorithms with dynamic population size and local exploration for multiobjective optimization. IEEE Transactions on Evolutionary Computation, 5(6), 565588.

14. Liao, X.; Li, Q.;Yang, X.; Zhang, W.; and Li, W.(2008). Multiobjective optimization for crash safety design of vehicles using stepwise regression model.Structural and Multidisciplinary Optimization, 35(6), 561-569.

15. Chankong, V.; and Haimes, Y.Y. (1983). Multiobjective decision making theory and methodology. New York: North-Holland.

\section{AUTHORS BIOGRAPHY}

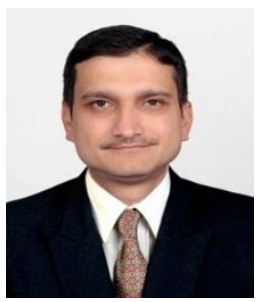

Mr. Shailesh Sharad Kadre received his M.Tech. Degree from the Department of Mechanical Engineering \& specialization in Machine Dynamics at IIT Kharagpur, India, in 1996. He is currently working as Assistant General Manager at Cyient Ltd, Hyderabad, India. He is pursuing his Ph.D. degree in Mechanical Engineering from College of Engineering Pune. His current research interest includes surrogate model based design optimization, Finite element analysis and test correlations.

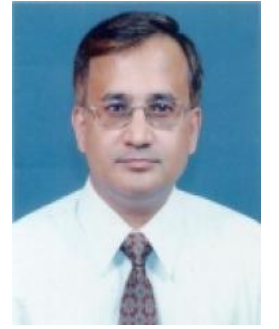

Dr.Vipin Kumar Tripathi received his Ph.D degree from the Department of Mechanical Engineering at IIT Kanpur, India. He is currently an associate professor and Prof in Charge, CAD/CAM/CAE Centre at college of Engineering Pune, India. He has a 20 pus years of teaching experience with numerous international publications. His research interest includes Knowledge in Optimization, CAD, Composite materials. 